\title{
Data-Driven Multi-Energy Investment and Management under Earthquakes
}

\author{
Pengfei Zhao, Chenghong Gu, Member, IEEE, Zhidong Cao, Yichen Shen, Fei Teng, Member, IEEE, Xinlei Chen, \\ Chenye Wu, Member, IEEE, Da Huo, Xu Xu, Member, IEEE and Shuangqi Li, Student Member, IEEE
}

\begin{abstract}
Seismic events can severely damage both electricity and natural gas systems, causing devastating consequences. Ensuring the secure and reliable operation of the integrated energy system (IES) is of high importance to avoid potential damage to the infrastructure and reduce economic losses. This paper proposes a new optimal two-stage optimization to enhance the reliability of IES planning and operation against seismic attacks. In the first stage, hardening investment on the IES is conducted, featuring preventive measures for seismic attacks. The second stage minimizes the expected operation cost of emergency response. The random seismic attack is modelled as uncertainty, which is realized after the first stage. An explicit damage assessment model is developed to define the budget set of the uncertain seismic activity. Based on the survivability of transmission lines and gas pipelines of IES, an optimal system investment plan is developed. The problem is formulated as a two-stage distributionally robust optimization (DRO) model, which is tested on an integrated IEEE 30-bus system and 20-node gas network. Case studies demonstrate that the twostage DRO outperforms robust optimization and a single-stage optimization model in terms of minimizing the investment cost and expected economic loss. This work can help system operators to make economical hardening and operation strategies to improve the reliability of IES under seismic attacks, thus managing a more robust and secure energy system.
\end{abstract}

Index Terms-Distributionally robust optimization, emergency response, integrated electricity and gas system, reliability.

This work was supported by the National Science Fund for Distinguished Young Scholars, No. 72025404. This work was supported by the National Natural Science Foundation of China (Nos. 72042018, 71621002).

P. Zhao and Z. Cao (corresponding author) are with the Institute of Automation, Chinese Academy of Sciences, Beijing, China and School of Artificial Intelligence, University of Chinese Academy of Sciences, Beijing, China. (email: P.Zhao0308@gmail.com, Zhidong.Cao@ia.ac.cn)

C.Gu, Y. Shen and S. Li are with the Department of Electronic \& Electrical Engineering, University of Bath, Bath, UK.(email: C.Gu@bath.ac.uk; Y. Shen@bath.ac.uk and S. Li@bath.ac.uk).

F. Teng is with the Department of Electrical and Electronic Engineering, Imperial College London, London SW7 2AZ, U.K. (e-mail: f.teng@imperial.ac.uk).

$\mathrm{X}$. Chen is with the Electrical Engineering Department, Carnegie Mellon University, Pittsburgh, USA. (email: xinlei.chen@sv.cmu.edu)

C. Wu is with the School of Science and Engineering, the Chinese University of Hong Kong, Shenzhen, Shenzhen, Guangdong 518172 China, and also with the Shenzhen Institute of Artificial Intelligence and Robotics for Society, Shenzhen, Guangdong 518129 China.

D. Huo is with the School of Engineering, Newcastle University, UK (email:Da.Huo@newcastle.ac.uk).

$\mathrm{X} . \mathrm{Xu}$ is with the Department of Electrical Engineering, The Hong Kong Polytechnic University, Hung Hom, Hong Kong Special Administrative Region, China. (email: benxx.xu@ connect.polyu.hk).

\section{INTRODUCTION}

$\mathrm{N}_{\mathrm{s}}^{\mathrm{A}}$ ATURAL disasters can cause huge power losses of energy systems that threaten the economy [1]. Earthquake is considered as one of the most disruptive natural disasters, which may cause large-scale blackouts without sufficient time for the response due to the weak predictability $[2,3]$. The Wenchuan earthquake in May 2008 damaged around 270 transmission lines and 900 substations, leaving 46 million people suffered without electricity. A massive power outage was caused in the 2010 Chile earthquake. Approximately 3GW generation capacity became unavailable, $26 \%$ of transmission network substations were damaged, where $93 \%$ of residents suffered from a power outage with two weeks [4]. According to existing research, more than $90 \%$ of such damage can be avoided if upgraded seismic preventive measures are adopted [5].

To enhance the reliability of power systems against natural disasters, reliability planning has been extensively investigated. Paper [6] proposes a multi-stage and multi-zone based robust optimization (RO) for a bi-level reliability problem considering power line hardening and distributed generation resource placement. Different grid enhancing strategies against extreme weather conditions are considered in [7] in a tri-level framework, which is transformed into an equivalent bi-level problem and solved by a greedy searching algorithm. Paper [8] develops a two-stage stochastic optimization (SO) for reliability planning in a large-scale transmission network to mitigate seismic risk. Optimal capacity expansion is considered as the planning strategy based on explicit damage distribution.

Existing research of seismic attack-based preventive measures mainly contains optimal system expansion and mobile energy storage supplement. To enhance the reliability and protect the system against seismic attacks, paper [8] designs a transmission and expansion scheme, where a SO-based approach is utilized for capturing the uncertain seismic attacks. In [9], mobile power sources are utilized as grid-support resources during seismic attacks for distribution systems. However, the capacity of mobile energy storage and electric vehicles is insufficient for hedging against seismic attacks for transmission systems. Paper [10] investigates the feasibility of energy storage utilization to improve system reliability against seismic attacks, which helps to supply critical loads during and following seismic attacks. Grid hardening, including constructing new lines and facilities, upgrading damaged poles and burying power lines underground, etc, is the most widelyproposed and effective measure to protect systems against 
natural disasters [11]. In our paper, reconstruction and upgrade to make systems resistant to earthquakes are modelled as the hardening measures for power lines and gas pipelines. It is assumed that the hardened lines will survive from seismic attacks $[6,8,12]$. The hardening strategy is considered in the first stage of the proposed hierarchical two-stage framework.

Meanwhile, energy infrastructures are becoming more complex and independent, especially with higher attention on the interdependence of different energy carriers. The rapid growth of gas consumption and mushrooming deployment of gas well and electrolysis have boosted the synergetic integration of electricity and natural gas systems. The integrated energy system (IES) can significantly increase energy utilization efficiency. Therefore, enhancing reliability to withstand seismic hazards and mitigate resulting damages is of great vitality for IES. A two-stage robust integrated planning of IES is proposed for enhancing the reliability in [13], which is implemented by replacing power lines by a gas transportation system. Paper [12] optimally minimizes the worst-case electricity and gas load shedding through a tri-level robust planning model with network hardening. Paper [14] proposes a reliability assessment for IES including heating, cooling and distributed generation systems. The proposed assessment is quantified through functionality loss and monetary costs.

However, recent IES planning studies consider the impacts of general natural disasters without specifying the types and ignore the attack assessment model, hardly targeting at mitigating seismic risks $[12,13]$. This is impractical as the impacts of natural disasters depend on the grid structure, disaster types, duration and intensity, etc. Different natural disasters could have a very different damage scale that needs specified preventive investment strategies. Thus, it is essential to develop models for assessing seismic risks on IES.

To accommodate the uncertainty of natural disasters, $\mathrm{RO}$ and SO have been extensively applied $[7,8,13]$. Nevertheless, RO ensures system robustness while inevitably leads to over conservativeness. SO requires explicit distributions with a large number of samples, which not only produces a high computational burden but also is not always practical. Distributionally robust optimization (DRO) bridges the strengths of RO and SO, which relaxes the assumption of specifying a certain distribution and considers the worst distributions compared to the worst-case oriented RO. An optimal gas-power flow is proposed in [15] by DRO with wind uncertainty. Wasserstein metric is used to select candidate distributions. A two-stage DRO model for IES scheduling is proposed in [16] and compared with traditional adjustable robust optimization, proving that DRO generates less conservative and more economical solutions.

This paper targets at alleviating the impacts of seismic events on both power lines and gas pipelines of IES. A two-stage DRO model is proposed to enhance the reliability for an IES, where the damage on both power lines and gas pipelines are considered. For simplicity, the keywords 'distributionally robust', 'seismic', 'integrated' and 'planning' are picked and this proposed model is referred to DR-SIP. The seismic activities are regarded as uncertain events and the random damage on power lines and pipelines are regarded as uncertainties, which are handled by DRO. The first stage minimizes the investment cost for hardening IES against seismic attacks. The hardening strategy incorporates strengthening power lines and gas pipelines with earthquakeresistant material and design. After the uncertainty of seismic activities is realized, the second stage minimizes the loss of emergency response through load shedding under the worst potential seismic risks. An improved Benders Decomposition is utilized to solve this IES reliability optimization problem, which is then demonstrated through the extensive case study. The merits of the proposed model are summarized in the case study section.

The main contributions of this paper are as follows:

1) A two-stage coordinated reliability enhancement and emergency response modelling incorporating both planning and operation schemes is developed to enhance IES reliability in the worst-distributed seismic attacks.

2) It develops a novel assessment model of IES performance against seismic attacks. This probabilistic quantification is estimated by damage scenarios, easily combined with the proposed two-stage DRO model. The coordinated assessment-optimization model enables the design of the optimal enhancement plans for IES.

3) It utilizes the novel DRO in IES reliability assessment and enhancement with a tractable reformulation. Historical information on seismic events is efficiently used to reduce solution conservativeness, thus producing more economical investment and operation decisions.

The rest of this paper is organized as follows. Section II proposes the damage assessment of seismic events. Section III designs reliability enhancement strategies. Section IV presents the mathematical formulation for reliability planning and emergency operation. The methodology and solution algorithm are given in section V. Section VI demonstrates case studies and the performance of DR-SIP. Conclusions are drawn in section VII.

\section{ASSESSMENT OF SEISMIC DAMAGE ON IES}

This section provides the seismic damage modelling for both electricity and natural gas systems, which mainly considers the damages on power lines and gas pipelines. The relationship between damage consequence and seismic level is established in this section. The seismic intensity is described by peak ground acceleration (PGA) and peak ground velocity (PGV), which are mainly related to landslides and surface faulting [17]. The relationship between seismic level, PGA and PGV are summarized in TABLE I $[18,19]$.

The concept of connection loss (CL) is adopted to quantify the line failures of electricity systems [20] due to the damage on pylons and conductors from seismic events. They destroy conductors and shake pylons and consequently damage power lines. For simplicity, this paper considers the number of damaged power lines, which are modelled as CL. Paper [21] concludes four-line damage states, minor, moderate, extensive and complete, which refers to $4 \%, 12 \%, 50 \%$ and $80 \%$ of CL, indicating the number of damaged power lines in percentage, which correspond to $P_{m i}, P_{m o}, P_{e x}$ and $P_{c o}$ in (1). 


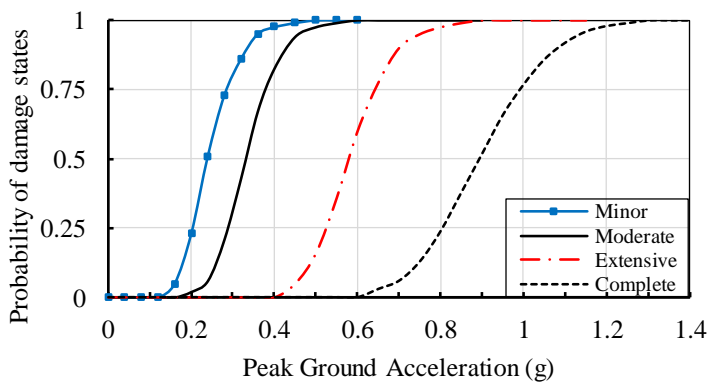

Fig. 1. Fragility curve of damage states for seismic attacks.

TABLE I

RANGES OF PGA, PGV AND SEISMIC INTENSITY

\begin{tabular}{|c|c|c|c|c|c|c|c|c|}
\hline Intensi & ty I & II $\sim$ III & IV & V & VI & VII & VIII & IX \\
\hline $\begin{array}{l}\text { PGA } \\
(\% g)\end{array}$ & $<0.17$ & $0.2-1.4$ & $1.4-3.9$ & $3.9-9.2$ & $9.2-18$ & $18-34$ & $34-65$ & $65-124$ \\
\hline $\begin{array}{l}\mathrm{PGV} \\
(\mathrm{cm} / \mathrm{s})\end{array}$ & $<0.1$ & $0.1-1.1$ & $1.1-3.4$ & $3.4-8.1$ & $8.1-16$ & $16-31$ & $31-60$ & $60-116$ \\
\hline
\end{tabular}

A random intensity level can cause a certain range of PGA, presented in TABLE I. Based on the fragility curve in Fig. 1, a random PGA can cause an earthquake in one or a combination of different line damage states. For example, when PGA is $0.6 \mathrm{~g}$, the line damage state of being complete is $0 \%$ and being extensive is $60 \%$, while $100 \%$ for being moderate and minor. Thus, the expected number of line failures modelled as CL under a specific PGA is described in (1).

$$
\begin{aligned}
& N_{E D}=P_{c o} C L_{c o}+\left(P_{e x}-P_{c o}\right) C L_{e x} \\
+ & \left(P_{m o}-P_{e x}\right) C L_{m o}+\left(P_{m i}-P_{m o}\right) C L_{m i}
\end{aligned}
$$

The steps to specify seismic damages on electricity lines are summarized as:

1. A random seismic intensity level is sampled from the Monte Carlo approach based on an empirical probability density function of seismic intensity level.

2. Based on TABLE I and the sampled intensity level, a certain range of PGA is given. Then Monte Carlo approach is used to pick a random PGA from the PGA range.

3. Based on the fragility curve in Fig. 1 and obtained PGA, the probability of 4 damage states is obtained.

4. Finally, according to equation (1), the expected number of damaged power lines, $N_{E D}$, is obtained.

To quantify the damage of the natural gas system due to seismic events, similar to CL of the electricity system, failure rate $(F R)$ is defined to represent the number of damage points on gas pipelines [22]. For simplicity, the natural gas system is assumed to operate in the steady-state, ignoring the dynamic gas leakage characteristics of pipelines.

$F R$ can be described with PGV:

$$
F R=\gamma(P G V)^{2.25} \mathrm{~L}
$$

The steps to acquire $F R$ of gas pipelines are as follows:

1. As described in step 1 of section A, the same seismic intensity level is obtained.

2. Based on TABLE I and obtained seismic intensity level, a certain range of PGV is given. Then Monte Carlo approach is used to pick a random $\mathrm{PGV}$ from the $\mathrm{PGV}$ range.

3. According to equation (2), FR is obtained.

\section{Mathematical Formulations For Reliability ENHANCEMENT}

Reliability planning and emergency response modelling are presented in this section. To begin with, the proposed reliability enhancement strategy is illustrated. The seismic hardening strategy is considered for power lines and gas pipelines. Linearized DC power flow and Weymouth gas flow equations are employed with load shedding to meet flow constraints. Finally, a two-stage optimization is proposed to minimize hardening costs in the first stage and operation cost in the second stage.

\section{A. Illustration of the Proposed Reliability Enhancement}

The proposed reliability-based IES planning consists of adaptation and recovery. The scope of this paper is on reliability planning and emergency response against seismic attacks. The preventive response is ignored as the preventive time after detecting seismic events is only up to a few seconds, which is too short for seismic preventive actions. In reliability planning, system operators make investment plans for seismic hardening on existing energy infrastructures. After the damage caused by seismic disasters, emergency response is implemented by system operators to mitigate the impact. There are two steps in the proposed DR-SIP: the first stage - reliability planning and the second stage - emergency response.

In the first stage, hardening on power lines is considered as the proposed reliability planning measure. In the second stage, the daily operation is implemented in the pre-hardened IES. To maintain the system balance while suffered from uncertain seismic attacks, load shedding is considered.

\section{B. Overall Objective}

The first and second-stage objectives are in (3) and (4). In (3), the objective $\Gamma_{P}$ represents total hardening investment cost for power lines and pipelines, which are given in the first and second terms, respectively, i.e., $\sum_{i, j \in I} h_{i j} \pi_{i j}$ and $\sum_{m, n \in M} h_{m n} \pi_{m n}$.

In (4), the objective $\Gamma_{R}$ is to minimize the emergency response cost. The final term of (4) represents the generation cost of gas wells in the gas system. It is assumed that the system operator curtails electricity and gas load under the worstdistributed seismic attacks to mitigate damage. During real seismic attacks, partial load needs to be curtailed to ensure the overall system supply-load balance. Since seismic attacks cause the unavailability of power lines and pipelines, energy transmission is significantly affected. Apart from load shedding, the generation function is also considered in the emergency response for normal system operation. The first and second terms are the shedding costs of electricity and gas loads. The generation cost function of electricity generators is a quadratic function, which is shown from the third term to the fifth term.

$$
\begin{gathered}
\Gamma_{P}=\sum_{i, j \in I} h_{i j} \pi_{i j}+\sum_{m, n \in M} h_{m n} \pi_{m n} \\
\Gamma_{R}=\sum_{t \in T, d_{e} \in D_{E}}^{m} \delta_{d_{e}, t} \pi_{d_{e}}+\sum_{t \in T, d_{e} \in D_{E}} \delta_{d_{g}, t} \pi_{d_{g}}+ \\
\sum_{t \in T, g e \in G E} \lambda_{g e}^{a} P_{g e, t}^{2}+\lambda_{g e}^{b} P_{g e, t}+\lambda_{g e}^{c}+\sum_{t \in T, g g \in G G} \lambda_{g g} P_{g g, t}
\end{gathered}
$$


The overall objective of the proposed DR-SIP is to minimize the planning cost in the first stage and the emergency response cost in the second stage in (5).

$$
\min \Gamma_{P}+\sup E_{P}\left[\Gamma_{R}\right]
$$

\section{Seismic Risk Oriented Reliability Planning}

Here, the feasibility set of hardening strategies for electricity network, gas network and the overall IES are shown in (6) to (8) respectively. The maximum number of power lines and pipelines to be hardened are constrained by (6) and (7). Constraint (8) means the total hardening investment cannot exceed the maximum monetary budget.

$$
\begin{gathered}
\sum_{i, j \in I} h_{i j} \leq E L_{h} \\
\sum_{m, n \in M} h_{m n} \leq P L_{h} \\
\sum_{i, j \in I} h_{i j} \pi_{i j}+\sum_{m, n \in M} h_{m n} \pi_{m n} \leq I C_{m a x}
\end{gathered}
$$

\section{Emergency Response}

Seismic events can have disruptive damage on power lines and gas pipelines, where the damages are considered as uncertainty, represented by binary variables $d_{i j}$ and $d_{m n}$. In the second stage of DR-SIP, to mitigate the loss of seismic events, electricity and gas load shedding is identified and implemented accordingly to keep the system balance. The sub-objective is to minimize the cost of load shedding and generation as an emergency response. When considering the effects of hardening strategies against seismic damage, the availability of lines needs to be identified and multiplied with the power and gas flow. Reference [12] considers the availability as $1-\xi+\xi h$, but the term $\xi h$ is nonlinear. This paper presents the availability as $\xi+$ $h$ in (14) and (15), which is linear but may cause $\xi+h=2$ and thus leads to overloading on power lines. For instance, in (14), $\left(\xi_{i j}+h_{i j}\right) f_{i j, \max }$ can be $2 f_{i j, \max }$. Accordingly, additional constraints are added to ensure the original limits, i.e., $-f_{i j, \max } \leq f_{i j, t} \leq f_{i j, \max }$.

The DC linearized power flow and Weymouth gas flow are utilized for modelling power flow and gas flow respectively, which are shown in (9) and (10).

$$
\begin{gathered}
x_{i j} f_{i j, t}=\left(\theta_{i, t}-\theta_{j, t}\right) \\
f_{m n, t}\left|f_{m n, t}\right|=\gamma_{m n}\left(P r_{m, t}{ }^{2}-P r_{n, t}^{2}\right)
\end{gathered}
$$

However, this paper considers the availability of asset that will inevitably lead to the nonlinear term $(\xi+h)\left(\theta_{i, t}-\theta_{j, t}\right)$ and $(\xi+h)\left(P r_{m, t}{ }^{2}-P r_{n, t}{ }^{2}\right)$. This nonlinearity is linearized based on sufficiently large constants $M_{i j}$ and $M_{m n}$. Thus, the linearized DC power flow and Weymouth gas flow constraints are used in (11)-(12) and (22)-(23).

The constraints of electricity and gas well output are in (16) and (17). Equations (18) and (19) show the constraints for electricity and gas load shedding. Equation (20) shows the upper and lower bounds for the pressure square of pipelines. Weymouth gas flow is presented in (21) and (22). The power and gas balance constraints are in (23) and (24).

$$
\begin{gathered}
x_{i j} f_{i j, t} \leq\left(\theta_{i, t}-\theta_{j, t}\right)+\left(1-\xi_{i j}-h_{i j}\right) M_{i j} \\
x_{i j} f_{i j, t} \geq\left(\theta_{i, t}-\theta_{j, t}\right)-\left(1-\xi_{i j}-h_{i j}\right) M_{i j} \\
-\pi \leq \theta_{i / j, t} \leq \pi
\end{gathered}
$$

$$
\begin{gathered}
-\left(\xi_{i j}+h_{i j}\right) f_{i j, \max } \leq f_{i j, t} \leq\left(\xi_{i j}+h_{i j}\right) f_{i j, \max } \\
0 \leq f_{m n, t} \leq\left(\xi_{m n}+h_{m n}\right) f_{m n, m a x} \\
P_{g e, m i n} \leq P_{g e, t} \leq P_{g e, m a x} \\
P_{g g, \min } \leq P_{g g, t} \leq P_{g g, m a x} \\
0 \leq \delta_{d_{e}, t} \leq \delta_{d_{e}, \max } \\
0 \leq \delta_{d_{g}, t} \leq \delta_{d_{g}, \max } \\
\operatorname{Pr}_{\min }{ }^{2} \leq P r_{t}{ }^{2} \leq P r_{\max }{ }^{2} \\
f_{m n, t} \leq \gamma_{m n}\left(\left(P r_{m, t}{ }^{2}-P r_{n, t}{ }^{2}\right)+\left(1-\xi_{m n}-h_{m n}\right) M_{m n}\right) \\
f_{m n, t} \geq \gamma_{m n}\left(\left(P r_{m, t}{ }^{2}-P r_{n, t}{ }^{2}\right)-\left(1-\xi_{m n}-h_{m n}\right) M_{m n}\right) \\
\sum_{d_{e} \in D_{E}} P_{d_{e}, t}-\delta_{d_{e}, t}=\sum_{g e \in G_{E}} P_{g e, t}+\sum_{i j \in I(t e r)} f_{i j, t}-\sum_{i j \in I(i n i)} f_{i j, t} \\
\sum_{d_{g} \in D_{G}} P_{d_{g}, t}-\delta_{d_{g}, t}+\sum_{g g \in G_{G}} P_{g g, t}= \\
\sum_{m n \in M(t e r)} f_{m n, t}-\sum_{g \in M(i n i)} f_{m n, t}
\end{gathered}
$$

The Weymouth gas flow is nonlinear, but $P r_{t}{ }^{2}$ is modelled in the squared form in this paper, which does not require linearization. The nonlinear term $f_{m n, t}{ }^{2}$ can be linearized by piecewise linear approximation to convert DR-SIP into a MILP problem [23], which is presented as follows. $h\left(f_{m n, t}\right)$ represents the nonlinear function, $\Delta f_{m n, k}$ is the segment of gas pipeline $m n$, and $\chi_{m n, t, k}$ is the auxiliary continuous variable.

$$
\begin{gathered}
h\left(f_{m n, t}\right) \approx h\left(\Delta f_{m n, 1}\right) \\
+\sum_{k \in K}\left(h\left(\Delta f_{m n, k+1}\right)-h\left(\Delta f_{m n, k}\right)\right) \chi_{m n, t, k} \\
f_{m n, t}=\Delta f_{m n, 1}+\sum_{k \in K}\left(\Delta f_{m n, k+1}-\Delta f_{m n, k}\right) \chi_{m n, t, k} \\
0 \leq \chi_{m n, t, k} \leq 1
\end{gathered}
$$

\section{SOLUTION PROCEDURE}

This section provides the procedure for solving the DR-SIP problem. The abstract forms of matrices and vectors are used to describe the constraints and variables. The ambiguity set for characterizing the seismic damage is constructed with moment information. Finally, the dual formulation is obtained and the proposed improved Benders Decomposition is used to solve the DR-SIP.

\section{A. Abstract Formulation}

For notation simplification, the compact form of the DR-SIP is given in (28)-(31). $x$ and $y$ are the first and second-stage decision variables. The uncertain seismic attack is represented by vector $\xi$. Equation (28) is the overall objective function containing (3) and (4). Vector $c$ consists of the unit hardening cost coefficient $\pi_{i j}$ and $\pi_{m n}$. Equation (29) represents constraints (6)-(8), where vector $b$ contains the allowed number of hardened power lines and pipelines as well as the monetary investment limit. Equation (30) shows the second-stage objective function where vector $f$ corresponds to the coefficients of (4). Equation (31) represents the second-stage constraints shown in (11)-(27).

$$
\begin{gathered}
\min _{x \in X} c^{\prime} x+\sup _{P \in D} E_{P}[Q(x, \xi)] \\
\text { s.t. } A x \leq b, \\
Q(x, \xi)=\min _{y} f^{\prime} y
\end{gathered}
$$




$$
\text { s.t. } E x+F y+G \xi \leq h,
$$

\section{B. Distributionally Robust Optimization}

The construction of the ambiguity sets for uncertain damage on both power lines and pipelines, i.e., $\xi_{i j}$ and $\xi_{i j}$, are proposed in this section. Moment information is widely used most for characterizing uncertainties and this paper utilizes the first moment for building ambiguity set. Since the damage probability can be obtained from historical data, thus $\mu_{E L}$ and $\mu_{P L}$ representing the estimated damage probability of power lines and pipelines, are used as the mean vectors. Compared with paper [24] which incorporates mean the vector and covariance matrix in the ambiguity set, this paper only utilizes the mean vector due to the data limit of distributional information. However, this paper provides a practical guide for characterizing uncertain rare events, such as natural disasters and contingency issues. The ambiguity sets are presented as follows.

$$
\left.\begin{array}{c}
D_{E L}=\left\{f\left(\xi_{i j}\right) \mid \begin{array}{c}
\mathrm{P}\left\{\xi_{i j} \in \Xi_{E L}\right\}=1 \\
\mathrm{E}\left\{\xi_{i j}\right\}=\mu_{E L}
\end{array}\right\} \\
D_{P L}=\left\{f\left(\xi_{m n}\right) \mid \begin{array}{c}
\mathrm{P}\left\{\xi_{m n} \in \Xi_{P L}\right\}=1 \\
\mathrm{E}\left\{\xi_{m n}\right\}=\mu_{P L}
\end{array}\right.
\end{array}\right\}
$$

As described in section II, the estimated damage level determines the number of damaged components. Accordingly, $\Xi_{E L}$ and $\Xi_{P L}$ are required as the support of seismic damage. The mean vector defined in equations (32) and (33) are obtained from $\frac{N_{E D}}{N_{p w r}}$ and $\frac{F R}{N_{g a s}}$, respectively, where $N_{p w r}$ and $N_{g a s}$ are the total number of power lines and pipelines in the proposed IES.

$$
\begin{aligned}
& \Xi_{E L}=\left\{\sum_{i, j \in I} \xi_{i j}=N_{E D}\right\} \\
& \Xi_{P L}=\left\{\sum_{m, n \in M} \xi_{m n}=F R\right\}
\end{aligned}
$$

The variables of the optimization problem are the probability densities in (32) and (33). The optimization problem contains a finite number of constraints and an infinite number of variables, which is non-tractable. By transforming it from the primal form into the dual form, the problem becomes tractable.

The second-stage objective function $\sup _{P \in D_{E L}, D_{P L}} E_{P}[Q(x, \xi)]$ can be represented by $S(x)$, where $P(\xi)$ is the probability density function.

$$
\begin{aligned}
S(x)^{\text {primal }}= & \max _{P(\xi) \in D_{E L}, D_{P L}} \int_{\Xi} Q(x, \xi) P(\xi) d \xi \\
\text { s.t. } P(\xi) \geq 0, \forall \xi \in \Xi & \\
& \int_{\Xi} P(\xi) d \xi=1 \\
& \int_{\Xi} \xi P(\xi) d \xi=\mu
\end{aligned}
$$

Based on dual theory [25], when the strong duality holds, the dual form of (36) is transformed to (40). Accordingly, the results of (40) are equal to those of $(36)[26,27]$. Now, the dual form has an infinite number of constraints and a finite number of variables after the dual formulation, which is easier to solve. The dual variables $\Psi$ and $\psi_{0}$ are associated with constraints (38) and (39).

$$
S(x)^{d u a l}=\min _{\Psi, \psi_{0}} \Psi \mu+\psi_{0}
$$

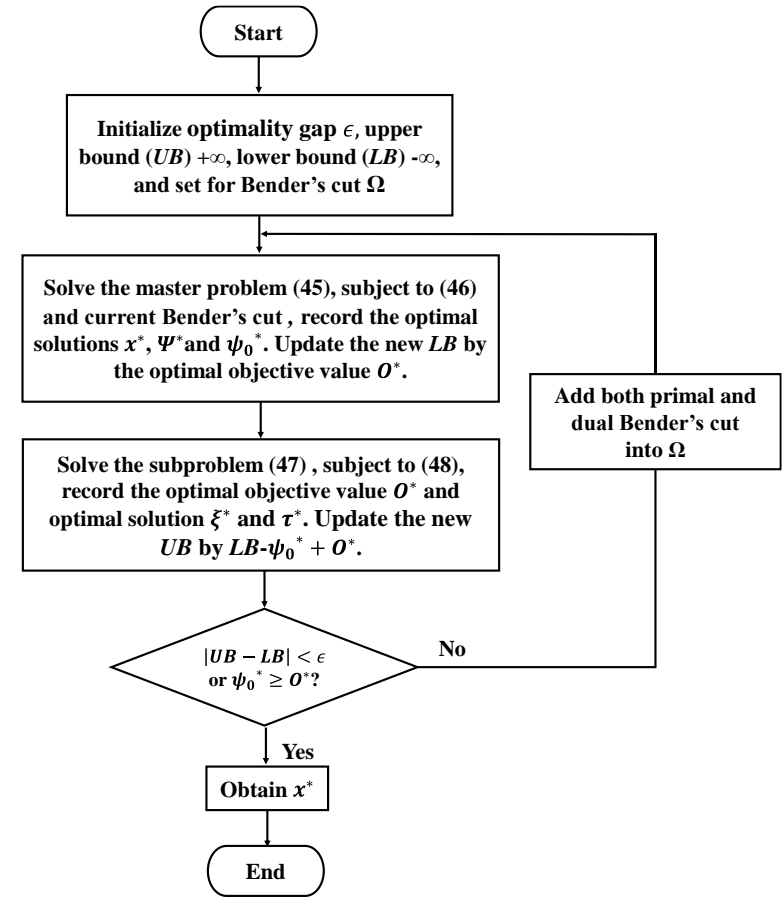

Fig. 2. Flow chart of Benders Decomposition method.

$$
\begin{gathered}
\text { s.t. } \Psi \xi+\psi_{0} \geq Q(x, \xi) \\
\forall \xi \in \Xi
\end{gathered}
$$

Then, the dual formulation (40) is substituted into (28) and the following reformulations can be obtained:

$$
\begin{gathered}
\min _{\Psi, \psi_{0}} c^{\prime} x+\Psi \mu+\psi_{0} \\
\text { s.t. } A x \leq b, \\
\Psi \xi+\psi_{0} \geq Q(x, \xi), \forall \xi \in \Xi
\end{gathered}
$$

\section{Improved Benders Decomposition}

An improved Benders Decomposition is applied to solve the DR-SIP in a master-subproblem framework, summarized in the flowchart in Fig. 2. Under the acceptable optimality gap, the master problem and subproblem are solved separately with the update of upper and lower bounds. Equation (44) is regarded as the Benders cut with respect to $\xi$. A Benders cut is formulated and added to the set in each iteration. Firstly, equations (42)-(44) are relaxed and only a subset of Benders cut is incorporated. The set of Benders cut becomes larger until the real gap between lower and upper bounds is smaller than the optimality gap. The master problem and primal subproblem are shown in (45)-(46) and (47)-(48).

$$
\begin{gathered}
\min _{\Psi, \psi_{0}} c^{\prime} x+\Psi \mu+\psi_{0} \\
\text { s.t. } A x \leq b, \\
\max _{\Psi} Q\left(x^{*}, \xi\right)-\Psi^{*} \xi \\
\text { s.t. } E x+F y+G \xi \leq h,
\end{gathered}
$$

The Benders Decomposition framework is given in Fig. 2. In addition, the approaching of optimality gap is enhanced through separating the Benders cut into primal and dual forms. To begin with, the new dual variable $\tau$ is introduced to represent the dual form of (30). Accordingly, (30) and (31) can be represented in the following closed form: 


$$
\begin{gathered}
\max \tau^{\prime}\left(h-E x-G \xi_{s}\right) \\
\text { s.t. } F^{\prime} \tau \leq f, \tau \geq 0
\end{gathered}
$$

Equation (47) is recast as (51) based on the new dual variable $\tau$.

$$
\begin{gathered}
\max \tau^{\prime}\left(h-E x-G \xi_{s}\right)-\Psi^{*} \xi \\
\text { s.t. } F^{\prime} \tau \leq f, \tau \geq 0
\end{gathered}
$$

It is worth noting that the term $\Psi^{*} \xi$ in (51) is nonlinear because it contains the product of dual variable $\Psi^{*}$ and binary variable $\xi$. Although it can be solved by some nonlinear solvers, linearization is still required to ensure a more efficient and global solution. McCormick inequality is used to relax this nonconvex problem, where $\vartheta_{i j}$ is used to represent $\tau_{i} G_{i j} \xi_{j}$.

$$
\begin{gathered}
\vartheta_{i j} \geq G_{i j} \tau_{i}-M\left(1-\xi_{j}\right) \\
\vartheta_{i j} \geq-M \xi_{j}
\end{gathered}
$$

Therefore, the dual subproblem can be written as:

$$
\begin{gathered}
\max \tau^{\prime}(h-E x)-\vartheta-\Psi^{*} \xi \\
\text { s.t. } F^{\prime} \tau \leq f, \tau \geq 0 \\
\vartheta_{i j} \geq G_{i j} \tau_{i}-M\left(1-\xi_{j}\right) \\
\vartheta_{i j} \geq-M \xi_{j}
\end{gathered}
$$

The primal Benders cut is based on the primal formulation of (30) and (31), which means at each iteration, the addition of primal Benders cut yields a new set of $y^{*}$ with respect of $\xi^{*}$. The primal form of steps 3 and 5 of Benders framework are shown below:

Step-3-primal: Solve the subproblem (55)-(58), record the optimal solution $\xi^{*}$ and the optimal objective value $O^{*}$. Set $U B=L B-\psi_{0}{ }^{*}+O^{*}$.

Step-5-primal: Add Benders cut:

$$
\begin{gathered}
E x+F y^{*}+G \xi^{*} \leq h, \\
\psi_{0} \geq f^{\prime} y^{*}-\Psi \xi^{*}
\end{gathered}
$$

To obtain the dual Benders cut, the dual form of (44) is given in (61). The dual form of steps 3 and 5 of Benders framework are summarized as:

Step-3-dual: Solve the dual subproblem (55)-(58), record the optimal solution $\xi^{*}$ and $\tau^{*}$ and the optimal objective value $O^{*}$. Set $U B=L B-\psi_{0}{ }^{*}+O^{*}$.

Step-5-dual: Add Benders cut:

$$
\psi_{0} \geq \tau^{\prime *}\left(h-E x-G \xi^{*}\right)-\Psi \xi^{*}
$$

\section{CASE STUdies}

This section presents the numerical case studies of the proposed DR-SIP on an integrated electricity and gas system. The IES consists of the standard IEEE 30 busbars electricity system and a 20-node gas network, shown in Fig. 3. The gas network includes 2 gas wells and 14 gas demands. A gas turbine is connected between bus 2 of the electricity network and node 2 of the gas network. The hardening investment cost is set as $\$ 4500$ and $\$ 5500$ for each power line and gas pipeline, respectively. The technical parameters of gas wells and electricity generators are given in TABLEs II and III. All numerical tests are executed on a personal computer with an Intel Core i7-8700 CPU with a 16 GB RAM. Coding is completed in MATLAB platform with MOSEK solver. Three cases are performed to optimize DR-SIP. The only difference between cases 2 and 1 is the addition of the planning stage before the seismic attacks.
TABLE II

PARAMETERS OF GAS WELLS

\begin{tabular}{cccc}
\hline \hline Node No. & $\begin{array}{c}P_{i_{g}, \max } \\
(\mathrm{kcf} / \mathrm{h})\end{array}$ & $\begin{array}{c}P_{i_{g}, \min } \\
(\mathrm{kcf} / \mathrm{h})\end{array}$ & $\lambda_{i_{g}}$ \\
\hline 1 & 3000 & 1000 & 2.2 \\
8 & 6000 & 1000 & 2 \\
\hline \hline
\end{tabular}

TABLE III

GENERATOR PARAMETERS

\begin{tabular}{ccccccc}
\hline \hline $\begin{array}{c}\text { Bus } \\
\text { No. }\end{array}$ & $\begin{array}{c}P_{i_{e}, \min } \\
(\mathrm{MW})\end{array}$ & $\begin{array}{c}P_{i_{e}, \max } \\
(\mathrm{MW})\end{array}$ & $\begin{array}{c}R_{i_{e}}^{+}, R_{i_{e}}^{-} \\
(\mathrm{MW})\end{array}$ & $a_{i_{e}}$ & $b_{i_{e}}$ & $c_{i_{e}}$ \\
\hline 1 & 50 & 200 & 20 & 0.004 & 2 & 6 \\
2 & 20 & 80 & 16 & 0.002 & 2 & 6 \\
5 & 15 & 50 & 10 & 0.006 & 1 & 8 \\
8 & 10 & 35 & 7 & 0.008 & 3 & 10 \\
11 & 10 & 30 & 10 & 0.025 & 3 & 18 \\
13 & 12 & 40 & 16 & 0.025 & 3 & 18 \\
\hline \hline
\end{tabular}

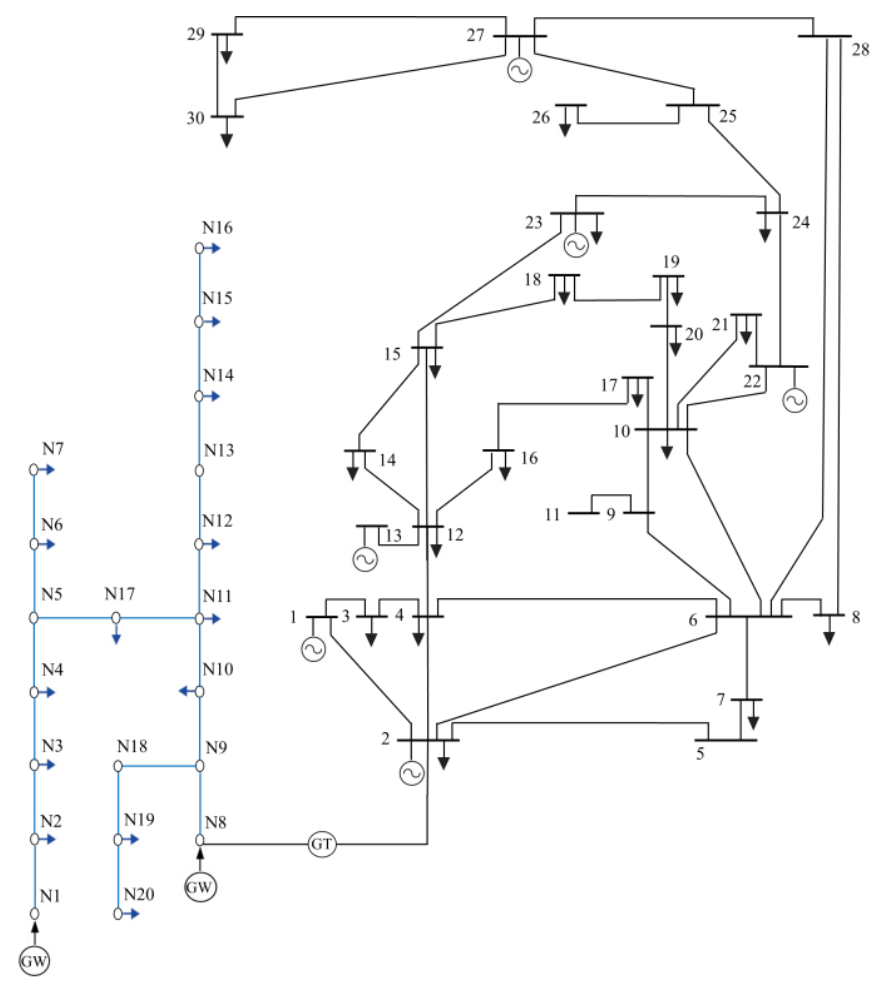

Fig. 3. Proposed test system.

- Case 1: Single-stage emergency response without considering hardening investment for IES.

- Case 2: Two-stage model including reliability enhancement planning and emergency response for IES.

- Case 3: Two-stage model for IEEE 30-bus system.

Section A presents comparisons of each case. Sections B and C illustrate the optimal hardening plan and computational results for case 2. Section D compares the numerical performance of case 2 between DRO and RO. Section E highlights the merits of the proposed model.

\section{A. Case Comparisons}

TABLE IV shows the operation and total cost for cases 1 and 2 with the increasing seismic intensity level. For case 1 , without hardening investment, the total cost is the same as the operation 
TABLE IV

COMPARISON BETWEEN CASE 1 AND 2

\begin{tabular}{ccccc}
\hline \hline & \multicolumn{2}{c}{ Case 1 } & \multicolumn{2}{c}{ Case 2 } \\
\cline { 2 - 5 } $\begin{array}{c}\text { Intensity } \\
\text { level }\end{array}$ & $\begin{array}{c}\text { Expected } \\
\text { operation } \\
\text { cost }\left(10^{3} \$\right)\end{array}$ & $\begin{array}{c}\text { Total } \\
\text { cost }\left(10^{3} \$\right)\end{array}$ & $\begin{array}{c}\text { Expected } \\
\text { operation } \\
\text { cost }\left(10^{3} \$\right)\end{array}$ & $\begin{array}{c}\text { Total } \\
\text { cost }\left(10^{3} \$\right)\end{array}$ \\
\hline I & 30 & 30 & 30 & 362 \\
II III & 30 & 30 & 30 & 362 \\
IV & 33 & 33 & 30 & 362 \\
V & 43 & 43 & 38 & 370 \\
VI & 56 & 56 & 42 & 374 \\
VII & 71 & 71 & 54 & 448 \\
VIII & 263 & 263 & 77 & 872 \\
IX & 402 & 402 & 125 & 920 \\
\hline \hline
\end{tabular}

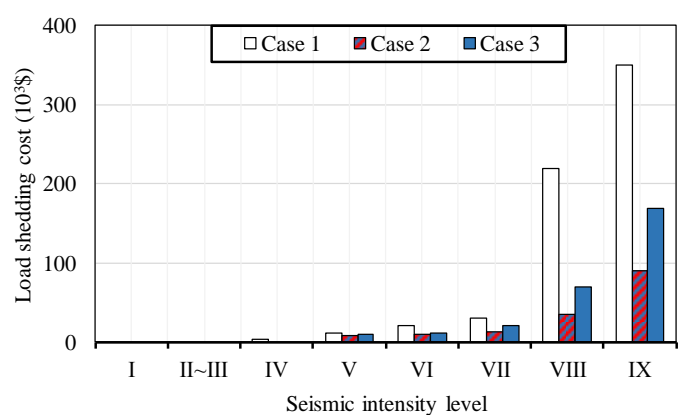

Fig. 4. Load shedding cost of three cases under different seismic intensity levels.

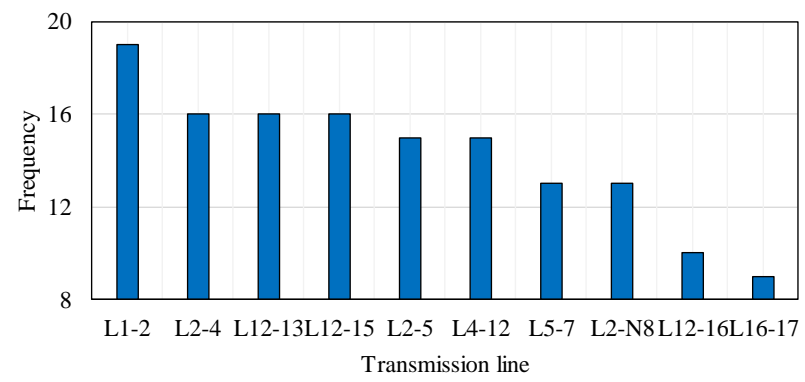

Fig. 5. The frequency of line hardened.

cost. For case 2, the total cost is the sum of operation and investment cost. The predefined investment budget for case 2 is 10 lines. When the intensity level is under level III, the expected line damage obtained from the seismic risk assessment model is around 0 , thus the operation cost for both cases 1 and 2 is the same as generation cost. When the intensity level is above III, operation cost increases since more load shedding is conducted. The investment cost of case 2 also increases from $332 \times 10^{3} \$$ to $795 \times 10^{3} \$$ even though the investment budget is fixed. The reason is that when more lines are damaged, DR-SIP invests on more important lines, which causes 139\% increase of investment cost when intensity is up to level IX. When the intensity is above level VII, the investment cost remains constant as the 10 most vital lines to be hardened are found.

It is to be noted that the operation cost in case 1 is always higher than that in case 2, i.e., the gap is from $10 \%$ under level IV to $220 \%$ under level IX, since more load shedding is made without hardening investment to protect lines. With the increasing intensity level, the total cost of case 1 is increasing faster than that of case 2 . When the intensity level is I, the total
TABLE V

OPTIMAL HARDENING PLAN UNDER DIFFERENT PLANNING BUDGET

\begin{tabular}{cc}
\hline \hline $\begin{array}{c}\text { Planning } \\
\text { budget }\end{array}$ & Optimal hardening plan \\
\hline 1 & $1-2$ \\
2 & $1-2,1-3$ \\
3 & $1-2,2-4,2-2-6,6-9$ \\
4 & $1-2,2-5,2-6,12-13,12-15$ \\
5 & $1-2,2-4,4-12,12-13,12-15,25-27$ \\
6 & $1-2,2-4,4-12,12-13,12-15,25-27,2-\mathrm{N} 8$ \\
7 & $1-2,2-4,2-5,5-7,4-12,12-13,12-15,8-28$ \\
8 & $1-2,2-4,2-5,5-7,4-12,12-13,12-15,8-28,2-\mathrm{N} 8$ \\
9 & $1-2,2-4,2-5,5-7,4-12,12-13,12-15,8-28,2-\mathrm{N} 8, \mathrm{~N} 8-\mathrm{N} 9$ \\
10 & $1-2,2-4,2-5,5-7,4-12,12-13,12-15,12-16,16-17,10-$ \\
15 & $17,10-21,27-30,8-28,2-\mathrm{N} 8, \mathrm{~N} 8-\mathrm{N} 9$ \\
& $1-2,2-5,2-6,4-6,6-7,6-10,4-12,12-13,12-15,10-21,10-$ \\
20 & $22,15-23,22-24,23-24,28-27,8-28,6-28,2-\mathrm{N} 8, \mathrm{~N} 8-\mathrm{N} 9$ \\
\hline \hline
\end{tabular}

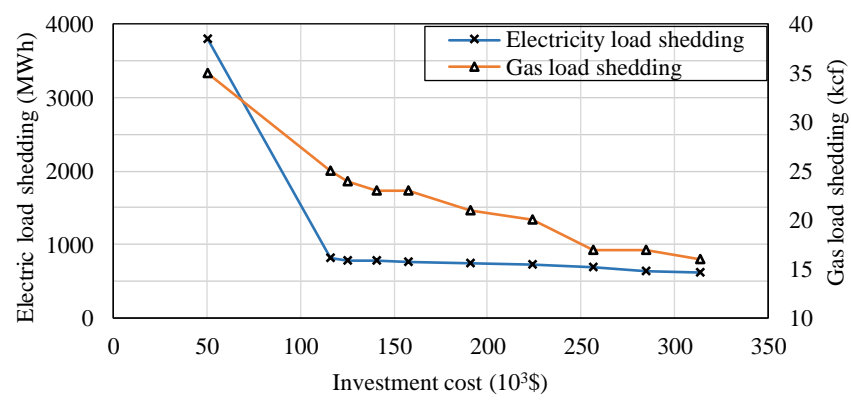

Fig. 6. Electricity and gas load shedding under different investment cost.

cost of case 1 is only $8 \%$ of case 2 , which reflects that only implementing load shedding without hardening investment is much more economical. However, under level IX, the ratio is up to $44 \%$, which shows that emergency response under seismic attacks without hardening planning causes huge economic loss.

When generation cannot satisfy all original load due to broken lines caused by seismic attacks, load shedding is made to maintain the system balance, which inevitably leads to huge load shedding cost. Fig. 4 depicts the load shedding cost for three cases under different seismic intensity levels. Higher load shedding cost is regarded as the system is more vulnerable when facing seismic attacks. It shows that case 1 yields the highest load shedding cost in all intensity levels, which reaches $350 \times 10^{3} \$$. This figure also shows that case 2 requires less load shedding than case 3 , with the cost in case $3: 169 \times 10^{3} \$$ and in case 2 : $90 \times 10^{3} \$$. The potential reason is that: i). Gas wells provide more supplies to the IES; ii) The IES network is more complex that can defend more severe seismic attacks; iii) Underground pipelines are more reliable than power lines, which is pre-set in the mean value vector in ambiguity set.

With increasing intensity levels, the cost difference between each case is becoming larger. When intensity level is IX, the cost of case 2 and 3 are only $26 \%$ and $48 \%$ of case 1 respectively. Overall, it can be observed that the integration of electricity and gas networks makes the system more reliable against seismic attacks.

\section{B. Optimal Hardening Plan}

The impact of increasing the budget on both planning and operation is given in TABLE V and the intensity level is set as 
TABLE VI

COMPUTATIONAL RESULTS UNDER DIFFERENT PLANNING BUDGET

\begin{tabular}{ccccc}
\hline \hline $\begin{array}{c}\text { Planning } \\
\text { Budget }\end{array}$ & $\begin{array}{c}\text { Investment } \\
\text { cost }\left(10^{3} \$\right)\end{array}$ & $\begin{array}{c}\text { Expected } \\
\text { operation } \\
\text { cost }\left(10^{3} \$\right)\end{array}$ & $\begin{array}{c}\text { Electricity } \\
\text { load shedding } \\
(\mathrm{MWh})\end{array}$ & $\begin{array}{c}\text { Gas load } \\
\text { shedding } \\
\left(\mathrm{Sm}^{3}\right)\end{array}$ \\
\hline 1 & 50 & 132 & 3800 & 35 \\
2 & 116 & 72 & 813 & 25 \\
3 & 125 & 72 & 787 & 24 \\
4 & 141 & 72 & 779 & 23 \\
5 & 158 & 72 & 763 & 23 \\
6 & 191 & 71 & 752 & 21 \\
7 & 224 & 71 & 727 & 20 \\
8 & 257 & 71 & 684 & 17 \\
9 & 285 & 65 & 640 & 17 \\
10 & 314 & 65 & 612 & 16 \\
15 & 446 & 62 & 429 & 12 \\
20 & 587 & 55 & 315 & 9 \\
\hline \hline
\end{tabular}

TABLE VII

COMPARISON WITH ROBUST OPTIMIZATION

\begin{tabular}{ccccc}
\hline \hline & \multicolumn{2}{c}{ Robust optimization } & \multicolumn{2}{c}{$\begin{array}{c}\text { Distributionally robust } \\
\text { optimization }\end{array}$} \\
\cline { 2 - 5 } Case & $\begin{array}{c}\text { Expected } \\
\text { operation } \\
\text { cost }\left(10^{3} \$\right)\end{array}$ & $\begin{array}{c}\text { Total } \\
\operatorname{cost}\left(10^{3} \$\right)\end{array}$ & $\begin{array}{c}\text { Expected } \\
\text { operation } \\
\text { cost }\left(10^{3} \$\right)\end{array}$ & $\begin{array}{c}\text { Total } \\
\operatorname{cost}\left(10^{3} \$\right)\end{array}$ \\
\hline 1 & 90 & 90 & 71 & 71 \\
2 & 72 & 495 & 54 & 448 \\
3 & 102 & 544 & 95 & 516 \\
\hline \hline
\end{tabular}

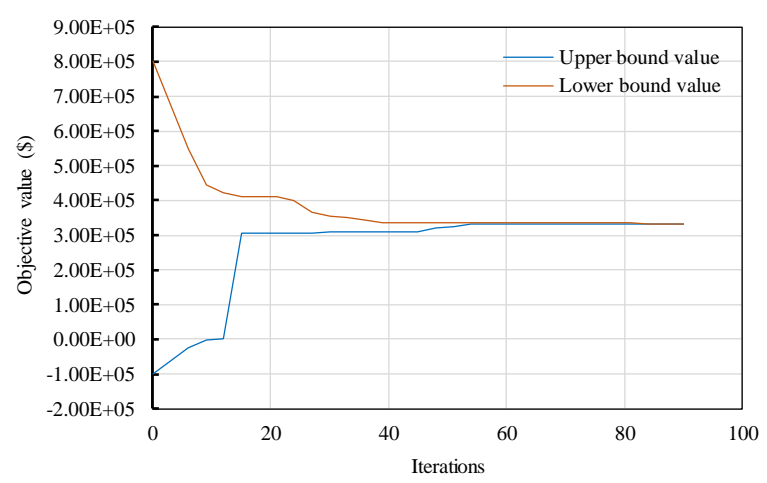

Fig. 7. Convergence rate of the Benders Decomposition method.

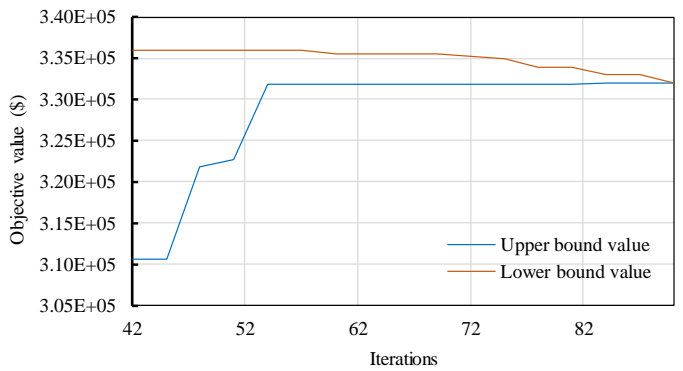

Fig. 8. Convergence result between 42th and 90th iterations.

TABLE VIII

COMPARISON BETWEEN TBD AND IBD

\begin{tabular}{cccc}
\hline \hline Method & $\begin{array}{c}\text { Investment } \\
\text { cost }\left(10^{3} \$\right)\end{array}$ & Iteration & $\begin{array}{c}\text { Computation } \\
\text { time }(\mathrm{hrs})\end{array}$ \\
\hline TBD & 330 & 145 & 3.8 \\
IBD & 332 & 90 & 2.1 \\
\hline \hline
\end{tabular}

level V. However, when a large number of damaged lines with few hardening lines considered, e.g., budget $=1$, may probably cause an infeasible solution. Since there are still many lines damaged without previously hardened, causing the congestion and unbalance among transmission lines. Line 1-2 is almost considered in all the budget ranges since both buses 1 and 2 contain the two largest generators and the power can be transmitted to buses 3, 5, 6 and gas bus 1. Line 2-4 is the second most frequent line to harden as the second largest generation of bus 2 needs to be securely transmitted to bus 4 , which is connected with buses 3,6 and 12. Fig. 5 shows the times for most frequent lines being hardened when the planning budget is under 20. It is concluded that buses 2 and 12 are the most significant buses that involved most frequently. As observed in Fig. 3, bus 2 connects with 5 buses with a generator and load. Bus 12 connects with 5 buses with a load.

\section{Computational Results Under Different Planning Budgets}

In TABLE VI, it shows that a larger planning budget directly leads to more investment cost, but also improves the reliability and accordingly reduces load shedding cost. When the budget increases from 1 to 2 , the investment cost increases dramatically by $66 \times 10^{3} \$$. The reason is that when the budget is extremely insufficient, e.g., budget $=1$ or 2 , the two most important lines 1 2 and 1-3 are chosen. Lines 1-2 and 1-3 are not only connected with large generators but also have large line ratings, and thus the hardening cost is high. Due to the hardening of lines 1-3, it survives from seismic attack by protecting 2980MWh electricity load and $10 \mathrm{Sm}^{3}$ gas load from shedding. When the budget increases above 2 , the investment cost and load shedding change slowly. Fig. 6 shows the load shedding curve for both electricity and gas with respect to different investment cost. When the investment cost increases, both electricity and gas load shedding decreases. The additional investment cost increasing from $50 \times 10^{3} \$$ to $116 \times 10^{3} \$$ helps to prevent load shedding greatly for both the electricity and gas networks. When the investment cost is above $116 \times 10^{3} \$$, the impact on load shedding is less effective.

\section{Comparison with Robust Optimization}

$\mathrm{RO}$ as a benchmark method is compared with the proposed DRO method in terms of mathematical performance in cases 1 , 2 and 3. From TABLE VII, it can be observed that RO yields higher costs in all the three cases. Overall, the total cost and operation cost of DRO are $13 \%$ and $18 \%$ less than those of RO respectively. The reason is that RO always considers line damages that cause the most severe load shedding. Therefore, in the first stage, RO makes decisions on hardening investment to protect the system before the potential worst damage. In the second stage, under the worst seismic attack, load shedding is made while the whole system flow balance is ensured.

\section{E. Computational Performance}

The proposed Benders Decomposition is denoted as improved Benders Decomposition (IBD) as it considers both primal and dual Benders cuts to accelerate the algorithm. The traditional Benders Decomposition is denoted as (TBD). In Figs. 7 and 8, the convergence result of IBD is given. Intensity level $\mathrm{V}$ is applied. It can be seen that 90 iterations are required for converging within the optimality gap. Eventually, the upper and 
lower bounds converge at $\$ 332000$. The comparison between IBD and TBD is given in TABLE VIII in terms of computational performance. The resulted investment costs are $\$ 330000$ and $\$ 332000$ under TBD and IBD, respectively. The proposed IBD significantly outperforms than TBD on computation time and convergence iteration times, which are 2.1 hours and 90 times. This result implies the improved computational efficiency of the proposed IBD.

\section{F. Discussion on Numerical Results}

Three cases are extensively investigated and the resulting difference shows that case 2 , i.e., the two-stage model including reliability enhancement planning and emergency response for IES, outperforms cases 1 and 3. Compared with case 1 which only contains the emergency response to maintain system power balance, case 2 provides a more secure and reliable operation scheme with $54 \%$ less load shedding cost. In comparison with case 3 which is implemented on the electricity system without gas system integration, case 2 produces better solutions with less load shedding and investment cost. Since the interdependency between electricity and gas systems enables coordinated energy flow to maintain both power and gas balance, it is effective to reduce load shedding and ensure the security of IES. The benefits of using the newly developed DRO over RO for all three cases are also analyzed, which results in reduced expected operation cost and total cost. The reason is that DRO captures the uncertainty of seismic attacks through partial distribution information of uncertainties via ambiguity sets, i.e., moment information. It considers more specific uncertainty information than RO and accordingly produces lessconservative results.

The extensive case study and analysis illustrate the advantages of DR-SIP, which are:

1) DR-SIP is a hybrid optimization model containing both planning and operation schemes. It not only provides an optimal hardening plan to enhance reliability against seismic attacks but takes actions to maintain supply and demand balance via load shedding, ensuring the security for IES in two stages.

2) The impact of seismic attacks on IES is assessed by considering historical data of seismic events in a probabilistic manner. Thus, the impact of seismic attacks can be easily included in the optimization model for reliability enhancement. 3) The developed two-stage DRO method is less conservative than RO because the statistical information of the uncertainty of seismic attacks is utilized.

4) This paper demonstrates that the integrated planning model can further improve the reliability of electricity systems, following the trend multi-vector energy system integration.

\section{CONCLUSION}

In this paper, a two-stage DRO method is developed to enhance the reliability of an IES under seismic attacks with combined planning and operation strategies. The proposed method provides optimal hardening plans for specific power lines and gas pipelines under different seismic intensity levels and investment budgets. Through extensive case study demonstrations, the key findings are as follows: i) In the first stage, DR-SIP effectively determines the most vital lines to harden. In the second stage, DR-SIP optimally shed loads in order to keep system balance and minimize the system operation cost; ii) With RO that considers the most extreme event and serious damage, the proposed DRO provides less conservative results for both planning and operation stages with $13 \%$ less cost; iii) Investment plan with a higher budget is more likely to yield a reliable IES with high-reliability performance; iv) The optimal hardening plan is effective for protecting transmission lines and loads and IES is more reliable than electricity network against seismic attacks.

This method can help system operators to make economical hardening and operation strategies to improve the reliability of integrated energy systems under seismic attacks.

\section{NOMENCLATURE}

\begin{tabular}{ccc}
\multicolumn{1}{c}{$A}$. & Sets & \\
$T$ & & Set for time periods. \\
$I$ & & Set for power lines. \\
$M$ & & Set for gas pipelines. \\
$G_{E}$ & & Set for electricity distribut \\
$G_{G}$ & Set for gas wells. \\
$D_{E}$ & Set for electricity loads. \\
$D_{G}$ & Set for gas loads. \\
& & B.
\end{tabular}

$C L_{c o}, C L_{e x}$, Connection loss for complete, extensive, $C L_{m o}, C L_{m i} \quad$ moderate and minor seismic level.

$\gamma \quad$ Failure rate constant for gas pipelines.

$\pi_{i j}, \pi_{m n} \quad$ Unit hardening cost for power lines and gas pipelines.

$E L_{h}, P L_{h} \quad$ Maximum number of hardening power lines and pipelines.

$I C_{\max } \quad$ Maximum monetary budget.

$x_{i j} \quad$ Reactance of power line ij.

$f_{i j, \max }$, Maximum power flow of line $\mathrm{ij}$ and gas flow

$f_{m n, \max } \quad$ of pipeline $\mathrm{mn}$.

$P_{g e, \min } \quad$ Minimum and maximum power output of

$P_{g e, \max } \quad$ electricity generators.

$\delta_{d_{e}, \max , \quad \text { Maximum limit for electricity and gas load }}$

$\delta_{d_{g}, \max } \quad$ shedding.

$P_{d_{e}, t}, P_{d_{g}, t} \quad$ Electricity and gas load demand at time t.

$P_{g g, \min } \quad \quad$ Minimum and maximum output of gas wells.

$P_{g g, \max }$

$P r_{\text {min }}, P r_{\text {max }} \quad$ Minimum and maximum pressure.

$\gamma_{m n} \quad$ Coefficient for Weymouth equation.

C. Variables

$P_{c o}, P_{e x}, P_{m o}, \quad$ Probability of being complete, extensive,

$P_{m i} \quad$ moderate and minor seismic attacks under a

$P_{E D} \quad$ Expected connection loss.

$h_{i j}, h_{m n} \quad$ Binary variables indicate if power line

$(\mathrm{i}, \mathrm{j}) /$ pipeline $(\mathrm{m}, \mathrm{n})$ is hardened.

$\theta_{i, t}, \theta_{j, t} \quad$ Phase angle at electricity bus $\mathrm{i}$ and $\mathrm{j}$

$x_{i j} \quad$ Reactance of power line ij.

$f_{i j, t}, f_{m n, t}, \quad$ Power and gas flow of power line ij and gas

$P_{g e, t}, P_{g g, t} \quad$ Electricity distributed generation and gas well

output at time t.

$\delta_{d_{e}, t}, \delta_{d_{g}, t} \quad$ Electricity and gas load shedding at time t.

$\mathrm{Pr}_{t} \quad$ Gas pressure at time t.

D. Uncertainty

$\xi_{i j}, \xi_{m n} \quad$ Binary variable indicates if power line $(\mathrm{i}, \mathrm{j}) /$ pipeline $(m, n)$ is damaged. 
$a_{i j}, a_{m n}$

Binary variable indicates if power line

$(\mathrm{i}, \mathrm{j}) /$ pipeline $(\mathrm{m}, \mathrm{n})$ is available.

\section{REFERENCES}

[1] D. N. Trakas and N. D. Hatziargyriou, "Optimal Distribution System Operation for Enhancing Resilience Against Wildfires," IEEE Transactions on Power Systems, vol. 33, no. 2, pp. 2260-2271, 2018, doi: 10.1109/TPWRS.2017.2733224.

[2] L. Tian, R.-s. Ma, H.-n. Li, and Y. Wang, "Progressive collapse of power transmission tower-line system under extremely strong earthquake excitations," International Journal of Structural Stability and Dynamics, vol. 16, no. 07, p. 1550030, 2016

[3] L. H. B. Haifeng, "State-of-the-art review on studies of disater resistance of highvoltage transmission tower-line systems," China Clvil Engineering Journal, no. 2, p. 8, 2007.

[4] J. C. Araneda, H. Rudnick, S. Mocarquer, and P. Miquel, "Lessons from the 2010 Chilean earthquake and its impact on electricity supply," in 2010 international conference on power system technology, 2010: IEEE, pp. 1-7.

[5] J. Eidinger, "Wenchuan earthquake impact to power systems," in TCLEE 2009: Lifeline Earthquake Engineering in a Multihazard Environment, 2009, pp. 1-12.

[6] W. Yuan, J. Wang, F. Qiu, C. Chen, C. Kang, and B. Zeng, "Robust OptimizationBased Resilient Distribution Network Planning Against Natural Disasters," IEEE Transactions on Smart Grid, vol. 7, no. 6, pp. 2817-2826, 2016, doi: 10.1109/TSG.2015.2513048.

[7] S. Ma, B. Chen, and Z. Wang, "Resilience Enhancement Strategy for Distribution Systems Under Extreme Weather Events," IEEE Transactions on Smart Grid, vol. 9, no. 2, pp. 1442-1451, 2018, doi: 10.1109/TSG.2016.2591885.

[8] N. R. Romero, L. K. Nozick, I. D. Dobson, N. Xu, and D. A. Jones, "Transmission and Generation Expansion to Mitigate Seismic Risk," IEEE Transactions on Power Systems, vol. 28, no. 4, pp. 3692-3701, 2013, doi: 10.1109/TPWRS.2013.2265853.

[9]Z. Yang, P. Dehghanian, and M. Nazemi, "Seismic-Resilient Electric Power Distribution Systems: Harnessing the Mobility of Power Sources," IEEE Transactions on Industry Applications, vol. 56, no. 3, pp. 2304-2313, 2020, doi: 10.1109/TIA.2020.2972854

[10] M. Nazemi, M. Moeini-Aghtaie, M. Fotuhi-Firuzabad, and P. Dehghanian, "Energy Storage Planning for Enhanced Resilience of Power Distribution Networks Against Earthquakes," IEEE Transactions on Sustainable Energy, vol. 11, no. 2, pp. 795-806, 2020, doi: 10.1109/TSTE.2019.2907613.

[11] P. Hoffman, W. Bryan, M. Farber-DeAnda, M. Cleaver, C. Lewandowski, and K. Young, "Hardening and Resiliency, US Energy Industry Response to Recent Hurricane Seasons," Office of Electricity Delivery and Energy Reliability, US Department of Energy, OE/ISER Final Report, 2010.

[12] C. He, C. Dai, L. Wu, and T. Liu, "Robust Network Hardening Strategy for Enhancing Resilience of Integrated Electricity and Natural Gas Distribution Systems Against Natural Disasters," IEEE Transactions on Power Systems, vol. 33, no. 5, pp. 5787-5798, 2018, doi: 10.1109/TPWRS.2018.2820383.

[13] C. Shao, M. Shahidehpour, X. Wang, X. Wang, and B. Wang, "Integrated Planning of Electricity and Natural Gas Transportation Systems for Enhancing the Power Grid Resilience," IEEE Transactions on Power Systems, vol. 32, no. 6, pp. 4418-4429, 2017, doi: 10.1109/TPWRS.2017.2672728.

[14] S. Moslehi and T. A. Reddy, "Sustainability of integrated energy systems: A performance-based resilience assessment methodology," Applied Energy, vol. 228, pp. 487-498, 2018/10/15/ 2018, doi: https://doi.org/10.1016/i.apenergy.2018.06.075.

[15] C. Wang, R. Gao, W. Wei, M. Shafie-khah, T. Bi, and J. P. S. Catalao, "Riskbased Distributionally Robust Optimal Gas-Power Flow With Wasserstein Distance," IEEE Transactions on Power Systems, pp. 1-1, 2018, doi: 10.1109/TPWRS.2018.2889942.

[16] Y. Zhang, J. Le, F. Zheng, Y. Zhang, and K. Liu, "Two-stage distributionally robust coordinated scheduling for gas-electricity integrated energy system considering wind power uncertainty and reserve capacity configuration," Renewable Energy, vol. 135, pp. 122-135, 2019/05/01/ 2019, doi: https://doi.org/10.1016/j.renene.2018.11.094.

[17] T. Datta, "Seismic response of buried pipelines: a state-of-the-art review," Nuclear Engineering and Design, vol. 192, no. 2-3, pp. 271-284, 1999.

[18] D. J. Wald, V. Quitoriano, T. H. Heaton, and H. Kanamori, Relationships between Peak Ground Acceleration, Peak Ground Velocity, and Modified Mercalli Intensity in California. 1999, pp. 557-564.

[19] W. Liu, Z. Li, Z. Song, and J. Li, "Seismic reliability evaluation of gas supply networks based on the probability density evolution method," Structural safety, vol. 70, pp. 21-34, 2018.

[20] K. Poljanšek, F. Bono, and E. Gutiérrez, GIS-based method to assess seismic vulnerability of interconnected infrastructure: A case of EU gas and electricity networks. Office for Official Publications of the European Communities, 2010.

[21] F. E. M. Agency, "HAZUS-MH multi-hazard loss estimation methodology, earthquake model, technical manual," ed: FEMA Washington, DC, 2003.
[22] G. Lanzano, E. Salzano, F. S. De Magistris, and G. Fabbrocino, "Seismic vulnerability of gas and liquid buried pipelines," Journal of Loss Prevention in the Process Industries, vol. 28, pp. 72-78, 2014.

[23] C. M. Correa-Posada and P. Sánchez-Martín, "Integrated Power and Natural Gas Model for Energy Adequacy in Short-Term Operation," IEEE Transactions on Power Systems, vol. 30, no. 6, pp. 3347-3355, 2015, doi: 10.1109/TPWRS.2014.2372013.

[24] W. Wei, F. Liu, and S. Mei, "Distributionally Robust Co-Optimization of Energy and Reserve Dispatch," IEEE Transactions on Sustainable Energy, vol. 7, no. 1, pp. 289-300, 2016, doi: 10.1109/TSTE.2015.2494010.

[25] E. Delage and Y. Ye, "Distributionally robust optimization under moment uncertainty with application to data-driven problems," Operations research, vol. 58, no. 3, pp. 595-612, 2010.

[26] D. Bertsimas, X. V. Doan, K. Natarajan, and C.-P. Teo, "Models for minimax stochastic linear optimization problems with risk aversion," Mathematics of Operations Research, vol. 35, no. 3, pp. 580-602, 2010.

[27] D. Bertsimas and I. Popescu, "Optimal inequalities in probability theory: A convex optimization approach," SIAM Journal on Optimization, vol. 15, no. 3, pp. 780-804, 2005.

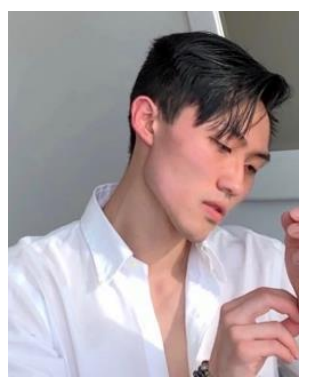

Pengfei Zhao (S'18) was born in Beijing, China. He received the double B.Eng. degree from the University of Bath, U.K., and North China Electric Power University, Baoding, China, in 2017. He received the Ph.D degree from the University of Bath, U.K. He was a visiting Ph.D. student at Smart Grid Operations and Optimization Laboratory (SGOOL), Tsinghua University, Beijing, China in 2019. Dr. Zhao is currently an Assistant Professor at the State Key Laboratory of Management and Control for Complex Systems, Institute of Automation, Chinese Academy of Sciences. His major research interests include the intelligent decision-making of complex energy systems and public health big data.

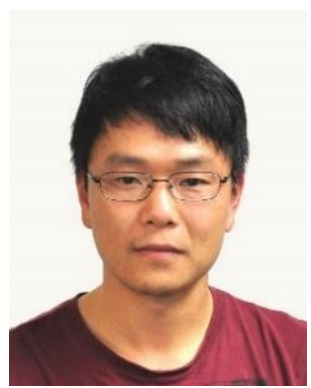

Chenghong Gu (M'14) was born in Anhui province, China. He received the Master's degree from the Shanghai Jiao Tong University, Shanghai, China, in 2007 in electrical engineering. He received the Ph.D. degree from the University of Bath, U.K. He is currently a Lecturer and EPSRC Fellow with the Department of Electronic and Electrical Engineering, University of Bath. His major research interest is in multi-vector energy system, smart grid, and power economics.

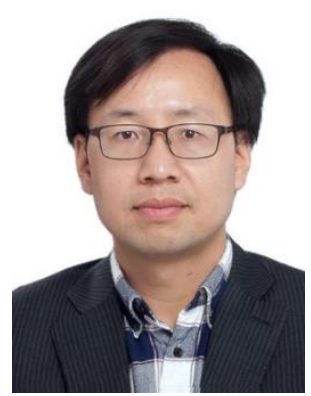

Zhidong Cao is an Associate Professor at the State Key Laboratory of Management and Control for Complex Systems, Institute of Automation, Chinese Academy of Sciences. His research interests include public health big data and infectious disease informatics. 


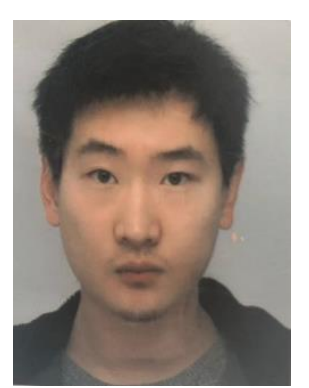

Yichen Shen was born in Hebei, China. He received the double bachelor degree in electrical engineering from North China Electric Power University, Baoding, China and University of Bath, U.K. in 2017. He is currently working towards the Ph.D. the University of Bath. His major research scope is resilient multi energy systems.

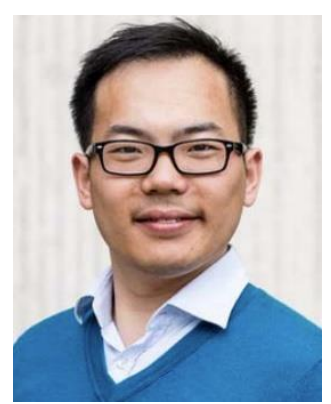

Fei Teng (Member, IEEE) received the B.Eng. in electrical engineering from Beihang University, Beijing, China, in 2009, and the Ph.D. degree in electrical engineering from Imperial College London, London, U.K., in 2015. He is currently a Lecturer with the Department of Electrical and Electronic Engineering, Imperial College London, U.K. His research focuses on scheduling and market design for low-inertia power system, cyber-resilient energy system operation and control, and objectivebased data analytics for future energy systems.

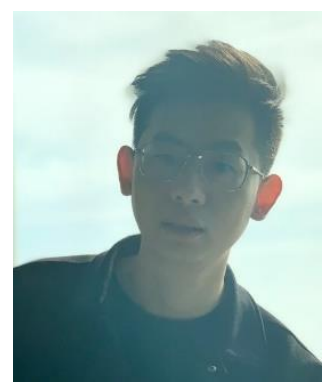

Xinlei Chen is currently a postdoctoral research associate in Electrical Engineering Department at Carnegie Mellon University. He received the B.E. and M.S. degrees in Electronic Engineering from Tsinghua University, China, in 2009 and 2012, respectively, and Ph.D degrees in Electrical Engineering from Carnegie Mellon University, Pittsburgh, PA, USA. His research interests lie in mobile computing, crowd intelligence, cyber physical system, mobile embedded system etc.

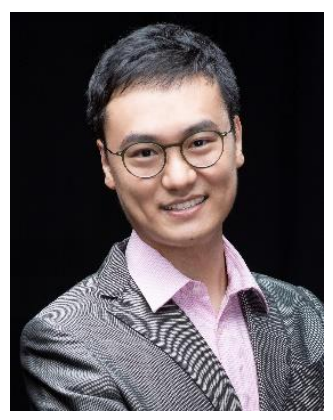

Chenye Wu (Member, IEEE) received the Ph.D degree from IIIS, Tsinghua University, in July 2013. His $\mathrm{Ph} . \mathrm{D}$. advisor is Professor Andrew Yao, the Laureate of the A. M. Turing Award in 2000. He is currently an Assistant Professor with the School of Science and Engineering, Chinese University of Hong Kong (CUHK Shenzhen), Shenzhen. Before joining CUHK Shenzhen, he was an Assistant Professor with IIIS, Tsinghua University. He worked with ETH Zurich as a Wiss. Mitarbeiter (Research Scientist), working with Prof. G. Hug, in 2016. Before that, Prof. K. Poolla and Prof. P. Varaiya hosted him as a Postdoctoral Researcher with UC Berkeley for two years. From 2013 to 2014 , he spent one year with Carnegie Mellon University as a Postdoctoral Fellow, hosted by Prof. G. Hug and Prof. S. Kar. He is currently working on economic analysis, optimal control, and operation of power systems. He was the Best Paper Award corecipients of IEEE SmartGridComm 2012, IEEE PES General Meeting 2013, and IEEE PES General Meeting 2020.

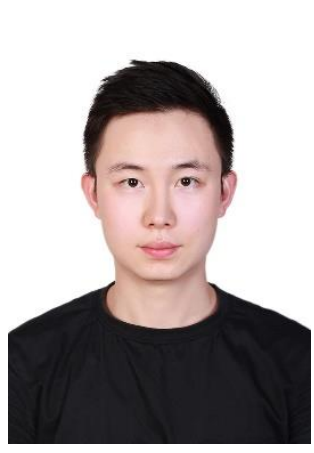

Xu Xu (S'18 M'19) received the M.E and Ph.D. degrees from The Hong Kong Polytechnic University, Hong Kong SAR in 2016 and 2019, respectively. Dr $\mathrm{Xu}$ is with the Department of Electrical and Electronic Engineering, The University of Hong Kong, Hong Kong SAR, China. His current research interests include power system planning and operation, renewable power integration, energy management, and artificial intelligence application in power engineering.

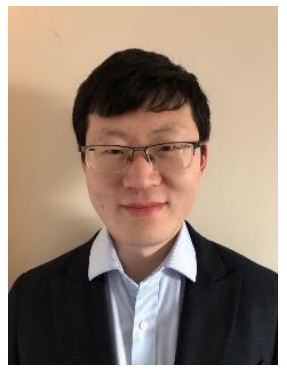

Da Huo was born in Inner Mongolia, China. He received the B.Eng. degrees in electrical and electronic engineering from the University of Bath, U.K., and in electrical power engineering from North China Electric Power University, Baoding, China, in 2014. He received the Ph.D. degree from the University of Bath, U.K, in 2018. He is currently a research associate with the School of Engineering, Newcastle University. His main research interests are multi-carrier energy system and smart grid.

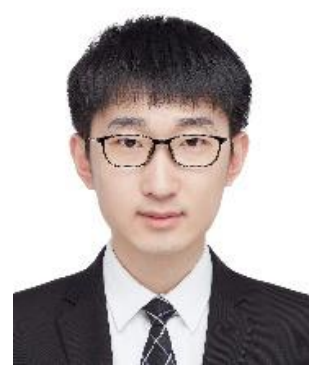

Shuangqi Li was born in Beijing, China. He received the B.Eng. degree in vehicle engineering from Beijing Institute of Technology, Beijing, China, in 2018 . He worked as a research assistant at the National Engineering Laboratory for Electric Vehicles, Beijing Institute of Technology from 2018 to 2019. Currently, he is pursuing the Ph.D. degree at the Department of Electronic and Electrical Engineering, University of Bath. His major research interest is the big data analysis, deep-learning algorithm, operation and planning of smart grid system and V2G service. 